\title{
PARAMUNNA FOLIACEA SP. N., ISOPODE ABYSSAL DE L'ATLANTIQUE TROPICAL ${ }^{1}$ )
}

\author{
PAR \\ PIERRE CHARDY \\ Centre Océanologique de Bretagne, B.P. 337, 29273 - Brest, France
}

Un prélèvement effectué sur la plaine abyssale du bassin de Guinée lors de la campagne „Walda” 2) du N.O. ,Jean Charcot”, a rapporté 3 exemplaires d'un Crustacé Isopode appartenant au genre Paramunna G. O. Sars, 1866. N'ayant pu les rattacher à aucune des espèces déjà connues de ce genre, nous avons été conduits à en faire une espèce nouvelle dont nous présentons la description dans cette note. -

\section{Paramunna foliacea sp. n. (figs. 1 et 2)}

Matériel étudié. - Campagne „Walda” - Station 33, prélèvement DS 25 (drague épibenthique, Hessler \& Sanders, 1967). $2^{\circ} 19,8^{\prime} \mathrm{N} 7^{\circ} 49,1^{\prime} \mathrm{E}$. Profondeur: $2470 \mathrm{~m}$. Trois spécimens 9 우. L'holotype est déposé dans la collection de Crustacés du Muséum national d'Histoire naturelle de Paris.

Description. - Le spécimen désigné comme holotype est une femelle adulte mesurant $2,5 \mathrm{~mm}$ de long et $1,2 \mathrm{~mm}$ de large (au niveau du 4ème péréionite). La forme générale du corps est large et ovale; sa silhouette très découpée est due au remarquable développement des processus latéraux des segments thoraciques (fig. 1A).

Céphalon. La partie antérieure de la tête se présente sous la forme d'un lobe rectangulaire dont le bord frontal est dépourvu de rostre. Les angles post-latéraux du céphalon sont allongés en lobes oculaires dépourvus d'yeux.

Péréion. Chaque segment thoracique possède une paire d'expansions latérales très développées, terminées par une petite épine. Le thorax, comprend 2 parties distinctes constituées chacune par des segments d'égale longueur. Les 4 premiers péréionites, constituant la partie antérieure, possèdent les processus latéraux larges et robustes tandis que les 3 derniers péréionites, beaucoup plus courts, possèdent des expansions latérales étroites et allongées. L'observation par transparence de l'extrémité latérale des péréionites, révèle l'existence d'une structure réticulée due à un système d'épaississement chitineux (fig. 1E).

Pléotelson plus long que large, composé de 2 segments: un pléonite vestigial réduit mais nettement visible dorsalement, et le telson, cordiforme, dont les bords latéraux sont dentés en scie.

1) Contribution $n^{\circ} 169$ du Département scientifique du Centre Océanologique de Bretagne.

2) Juin-Août 1971, Atlantique Sud. 
Antennule (fig. 2D). Premier article du pédoncule robuste, muni sur son bord interne d'un lobe saillant garni de 3 épines. Flagellum grêle, constitué de 4 articles dont le dernier porte une longue soie apicale rigide.

Antenne (fig. 1C) cassée au niveau du 4ème article du pédoncule (sur l'holo-

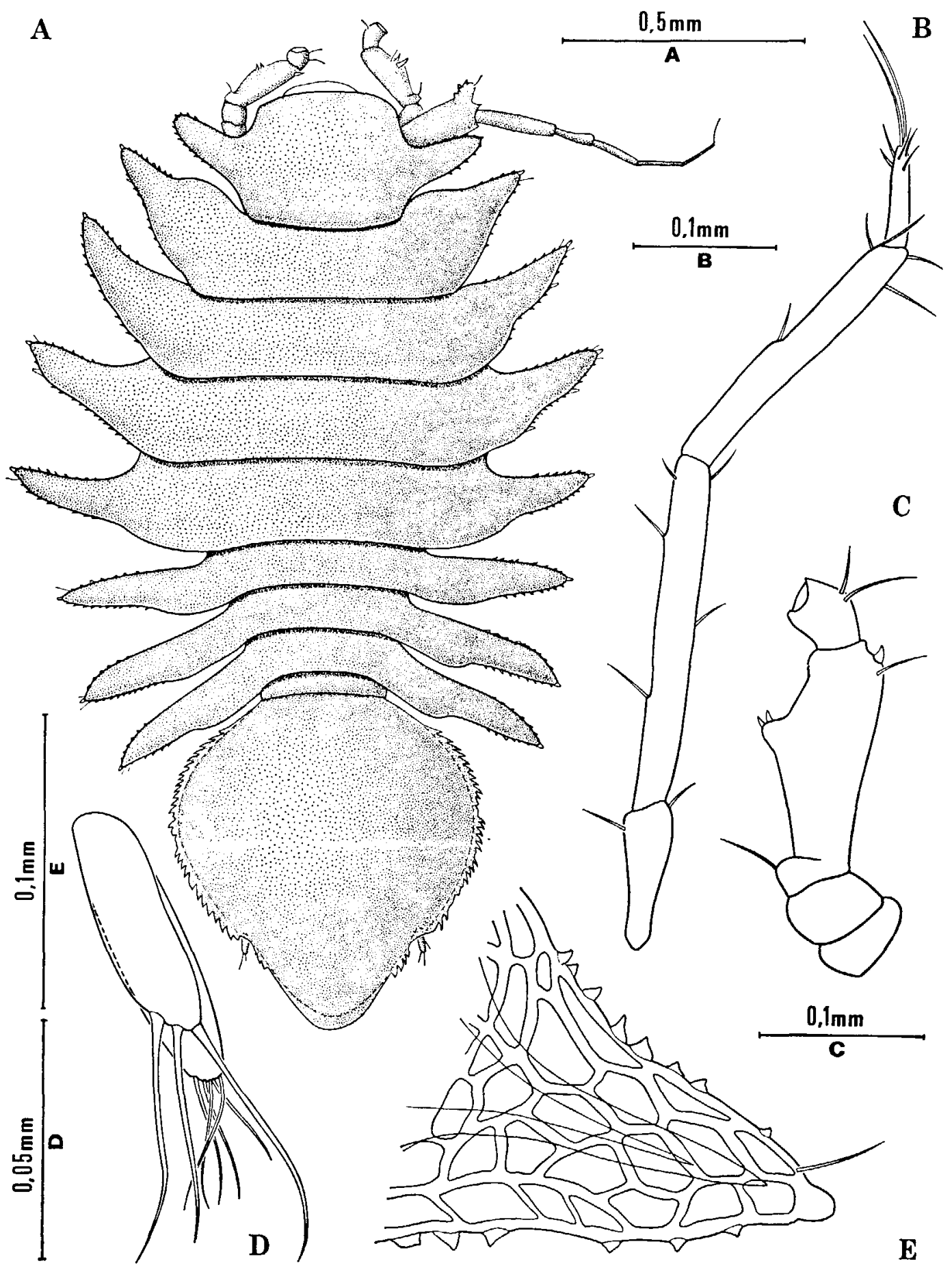

Fig. 1. Paramunna foliacea sp. n., holotype ( $\%$ ). A, vue dorsale de l'habitus; B, péréiopode 2; C, pédoncule de l'antenne; $\mathrm{D}$, vue ventrale de I'uropode; $\mathrm{E}$, détail d'un processus latéral (péréionite 1). 
type ainsi que sur les 2 autres spécimens récoltés). Le troisième article, très long, est armé de 2 tubercules épineux sur son bord interne.

Mandibule gauche (fig. 2B). Processus molaire robuste subcylindrique, caractéristique du sous-groupe des Munnini (créé par Hansen, 1916 et discuté en détail

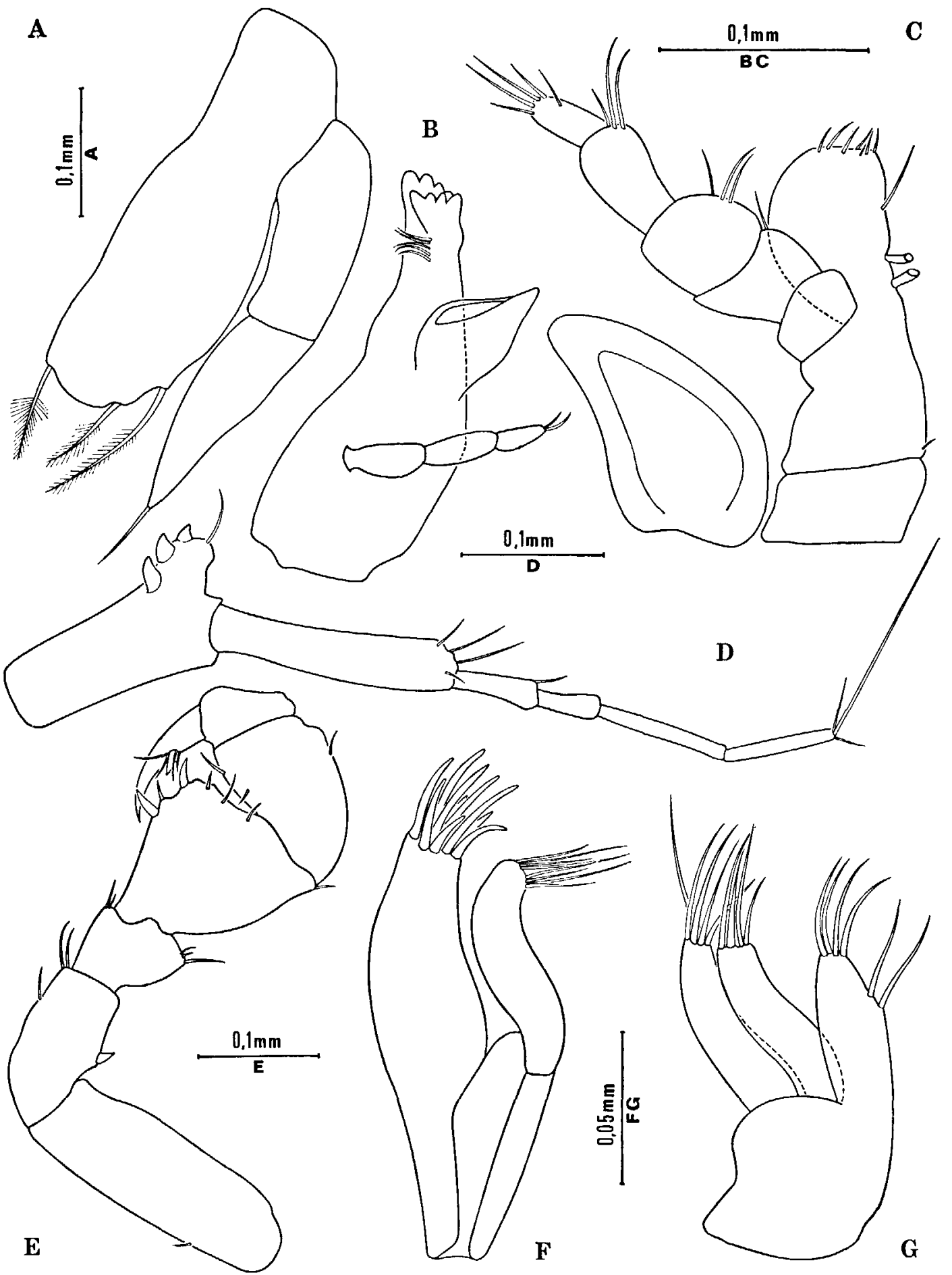

Fig. 2. Paramunna foliacea sp. n., holotype ( 9 ). A, 3ème pléopode; B, mandibule gauche; C, maxillipède; $\mathrm{D}$, antennule; $\mathrm{E}$, premier péréiopode; $\mathrm{F}$, maxillule; $\mathrm{G}$, maxille. 
par Wolff, 1962). Processus incisif étroit, muni d'une lacinia mobilis à 4 dents. Présence d'un palpe mandibulaire réduit constitué de trois articles sub-égaux.

Maxille (fig. $2 \mathrm{G}$ ) de type classique, sans particularité remarquable.

Maxillule (fig. 2F). Lobe inférieur bien développé dont la taille est sensiblement égale à celle du lobe supérieur.

Maxillipède (fig. 2C). Bord interne du basipodite porteur de 2 rétinacles. Les 3 premiers articles du palpe sont sensiblement plus larges que les 2 derniers. Epipodite court et arrondi à l'extrémité distale.

Premier péréiopode (fig. 2E) transformé en organe préhensile. Le carpopodite et le propodite, tous deux très épaissis, forment une forte pince terminée par un dactylopodite élargi armé d'une griffe longue et robuste.

Péréiopodes 2 à 7 (fig. 1B) tous identiques, constitués d'articles longs et grêles et terminés par une paire de longues soies souples.

Les pléopodes 1 et 2 sont soudés en un operculum sphérique chez la femelle. Le pléopode 3 (fig. 2A) est caractérisé par un exopodite relativement long, biarticulé, dont le dernier article est garni d'une simple soie.

Uropodes (fig. 1D) réduits, biramés, sans pédoncule, insérés latéralement sur le pléotelson. La rame interne, plus petite, est masquée dorsalement par la rame externe.

\section{DISCUSSION}

L'appartenance de Paramunna foliacea sp. n. à la famille des Munnidae (redéfinie par Wolff, 1962) est établie clairement par les caractères suivants: corps large et ovale; tête et tous les segments thoraciques libres; péréion divisé en 2 parties (péréionites 1-4 et péréionites 5-7); pléon plus large que long, trois premiers articles du palpe du maxillipède plus larges que les 2 derniers, péréiopode 1 préhensile.

Par ailleurs, l'absence de pédoncule à l'uropode, la partie distale arrondie de l'épipode et surtout la présence d'un processus molaire robuste et sub-cylindrique caractérisent le sous-groupe des Munnini.

Enfin, les plaques coxales non visibles dorsalement, la présence d'un palpe mandibulaire et l'absence d'épines dorsales sur le corps permettent de classer sans ambiguité l'espèce étudiée parmi les représentants du genre Paramunna G. $O$. Sars, 1866.

Paramunna foliacea sp. n. est caractérisée par le remarquable développement des processus latéraux du péréion et ne peut en aucun cas être confondue avec les autres espèces de ce genre.

Le genre Paramunna compte de nombreuses formes littorales; certaines d'entre elles étendent leur répartition verticale jusqu'au bathyal supérieur. Ainsi Paramunna rostrata Hodgson, 1910, considérée jusqu'à présent comme l'espèce la plus profonde au sein de ce genre, a été récoltée dans un intervalle bathymétrique compris entre 0 et $569 \mathrm{~m}$. Paramunna foliacea sp. n., prélevée sur les fonds du bassin de Guinée à $2400 \mathrm{~m}$ de profondeur, constitue donc l'unique représentant abyssal 
du genre Paramunna. A ce titre, l'absence d'yeux (présents chez la plupart des représentants du genre) et le développement des processus latéraux, peuvent être considérés comme des adaptations à la vie en profondeur. Par contre l'existence d'un pléonite distinct, constitue un caractère primitif. Paramunna foliacea $\mathrm{sp}$. n. possède donc une combinaison de caractères primitifs et évolués qui rend sa définition phylogénique, au sein de ce genre, particulièrement délicate.

\section{SUMMARY}

Description of a new species of isopod, Paramunna foliacea sp. n., collected from an abyssal station in the Guinea basin.

\section{RÉFÉRENCES BIBLIOGRAPHIQUES}

Hansen, H. J., 1916. Crustacea Malacostraca, 3. Danish Ingolf Exped., 3 (5): 1-262, pls. 1-16, 1 carte.

Hessler, R. \& H. Sanders, 1967. Faunal diversity in the deep-sea. Deep-Sea Res., 14: 65-78.

WOLFF, T., 1962. The systematics and biology of bathyal and abyssal Isopoda Asellota. Galathea Rep., 6: 1-315. 